\title{
Prevalence of airflow obstruction among ever-employed US adults aged 18-79 years by longest held occupation group: National Health and Nutrition Examination Survey 2007-2010
}

\author{
Laura Kurth, Brent Doney, and Cara Halldin \\ Respiratory Health Division, National Institute for Occupational Safety and Health, Centers for \\ Disease Control and Prevention, Morgantown, West Virginia, USA
}

\begin{abstract}
Objectives-To estimate the prevalence of spirometry-defined airflow obstruction among everemployed US adults.

Methods-Data from the 2007 to 2010 National Health and Nutrition Examination Survey (NHANES) for adults 18-79 years with valid spirometry and longest held occupation were analysed. The age-standardised prevalence of spirometry-defined airflow obstruction was estimated overall and by smoking status.
\end{abstract}

\begin{abstract}
Results-Age-standardised prevalence of airflow obstruction was $13.7 \%$ (95\% CI $12.4 \%$ to $15.0 \%)$ and was highest in participants aged $60-79$ years $(17.4 \%, 95 \%$ CI $15.2 \%$ to $19.6 \%)$, males (14.8\%, $95 \%$ CI $12.0 \%$ to $17.6 \%)$, non-Hispanic whites (15.4\%, 95\% CI $13.8 \%$ to $16.7 \%)$ and ever smokers $(19.1 \%, 95 \%$ CI $16.6 \%$ to $21.5 \%)$. Age-standardised prevalence of airflow obstruction was $>20 \%$ for installation, maintenance and repair occupations ( $\mathrm{p}=22.1 \%, 95 \% \mathrm{CI}$ $16.5 \%$ to $27.8 \%)$, and for construction and extraction occupations $(20.7 \%, 95 \%$ CI $13.5 \%$ to $27.9 \%)$.
\end{abstract}

Conclusions-Prevalence of airflow obstruction varied by demographic characteristics and occupational factors with a higher prevalence among ever smokers for most demographic characteristics and occupational factors. Study findings emphasise the importance of monitoring the lung function of workers in occupations with a high prevalence of airflow obstruction.

\footnotetext{
Correspondence to. Dr Laura Kurth, Respiratory Health Division, National Institute for Occupational Safety and Health, Centers for Disease Control and Prevention, 1095 Willowdale Road, Morgantown, WV, 26505, USA; vrz6@cdc.gov.

Contributors LK, BD and CH planned the study. LK analysed the data and submitted the study. LK and BD wrote the first draft. CH assisted in writing the introduction and discussion. All the authors provided comments on draft versions and approved the final version of the manuscript.

Disclaimer The findings and conclusions in this brief are those of the authors and do not necessarily represent the views of the National Institute for Occupational Safety and Health. Mention of a specific product or company does not constitute endorsement by the Centers for Disease Control and Prevention. This manuscript was prepared and written by NIOSH employees as part of their employment.

Competing interests None declared.

Data sharing statement We used publicly available data from the household interview survey and the standardised physical examinations conducted in mobile examination centres.
} 


\section{INTRODUCTION}

The overall age-adjusted prevalence of spirometry-defined airflow obstruction among US adults aged 40-79 years from 2007 to 2010 was $14.5 \%$ (airflow obstruction was defined per American Thoracic Society/European Respiratory Society (ATS/ERS) criterion as forced expiratory volume in the $1 \mathrm{~s}\left(\mathrm{FEV}_{1}\right)$ /forced vital capacity $(\mathrm{FVC})<$ lower limit of normal (LLN)). ${ }^{1}$ Airflow obstruction is a key feature of chronic obstructive pulmonary disease (COPD), asthma and other obstructive lung diseases. ${ }^{2}$ Smoking is a principal risk factor for airflow obstruction as it damages lung airways. ${ }^{3}$ Workplace exposures to vapours, gases, dust and fumes also increase the risk of obstructive lung disease. ${ }^{4}$ Results from the 1988 to 1994 National Health and Nutrition Examination Survey (NHANES) indicated that workers aged 30-75 years reporting a longest held job in the freight, stock and material handlers or armed forces occupation groups had higher odds of spirometry-defined COPD (FEV ${ }_{1} /$ $\mathrm{FVC}<70 \%$ and $\mathrm{FEV}_{1}<80 \%$ predicted) compared with office workers when analysing 14 occupation groups. ${ }^{5}$ Furthermore, an estimated $19.2 \%$ of COPD cases overall and $31.1 \%$ of COPD cases among never smokers were attributed to work. ${ }^{5}$ The purpose of this study was to estimate the prevalence of spirometry-defined airflow obstruction among the everemployed US adult population, using 2007-2010 NHANES data.

\section{METHODS}

The US population, 18-79 years, was studied using NHANES data from the combined cross-sectional 2007-2008 and 2009-2010 survey cycles. These were the most current NHANES cycles available with longest held occupation and spirometry data. The National Center for Health Statistics (NCHS) conducted NHANES, using a multistage probability sampling design to survey a representative sample of the civilian non-institutionalised US population. ${ }^{67}$ We used publicly available data from the household interview survey and the standardised physical examinations conducted in mobile examination centres (MEC). ${ }^{167}$ In the 2007-2010 NHANES, 11891 persons aged 18-79 years who provided interview data were eligible for the spirometry component of the physical examination. Of those, 1867 were excluded from spirometry for safety reasons, health reasons, or other reasons, and 501 had poor quality spirometry data. Spirometry details including eligibility criteria, spirometry procedures and spirometry quality are available elsewhere. ${ }^{7}$

NHANES demographic and risk factor variables analysed included age, gender, race/ ethnicity (including non-Hispanic white, non-Hispanic black, Mexican American, other Hispanic and other), occupational exposure, longest held occupation and cigarette use. Prior studies have examined obstructive lung diseases, including asthma and chronic bronchitis, attributed to occupation, in a range of populations including workers aged 18 years. ${ }^{4}$ Cigarette smoking status categories included never smokers and ever smokers. Never smokers were those 18-19 years who used neither tobacco nor nicotine products in the last 5 days and did not report using cigarettes in the past 5 days; and those 20-79 years who smoked <100 cigarettes during their entire life. Ever smokers were those aged 18-19 years who used cigarettes in the past 5 days and those aged 20-79 years who smoked at least 100 cigarettes during their entire life. Pack-years were estimated for ever smokers aged 20-79 
years by multiplying the number of packs of cigarettes smoked by the number of years reported smoking.

Participants reporting 'yes' to questions about ever having work exposure to mineral dust (ie, dust from rock, sand, concrete, coal, asbestos, silica, or soil), organic dust (ie, dust from flours, grains, wood, cotton, plants or animals), exhaust fumes (ie, fumes from trucks, buses, heavy machinery, or diesel engines), or other fumes (ie, vapours from paints, cleaning products, glues, solvents and acids; or welding/soldering fumes) in any job were considered to have 'occupational exposure'.

Longest held occupation was determined for ever-employed participants from the question, 'Thinking of all the paid jobs or businesses you ever had, what kind of work were you doing the longest?', or from the question related to current occupation ('what kind of work were you doing' in the past week) among participants reporting that their longest held occupation was also their current occupation. Participants not reporting a longest held occupation, such as participants who never worked, were excluded from the analysis. Longest held occupation was used since airflow obstruction often has a latency period. NCHS classified longest held occupation into 23 occupation groups based on 2002 Census Bureau Occupation Codes. ${ }^{7}$

The ATS/ERS criterion was used to define airflow obstruction $\left(\mathrm{FEV}_{1} / \mathrm{FVC}<\mathrm{LLN}\right)$ using lung function values from prebronchodilator spirometry. ${ }^{8}$ Normative reference equations developed from NHANES III data were used to determine the predicted and LLN pulmonary function values accounting for age, height, sex and race/ethnicity. ${ }^{9}$

\section{Data analysis}

Statistical analyses were performed using SAS V.9.3 (SAS Institute Inc, Cary, North Carolina, USA). Age-standardised prevalence of airflow obstruction with corresponding 95\% CIs was calculated using the standard age distribution of the 2000 US Census Population age structure for age groups $18-39,40-59,60-79$ years. ${ }^{6}$ NCHS MEC weights accounted for the sampling strategy (the year and sampling unit) to obtain unbiased, nationally representative prevalence estimates. ${ }^{6} \mathrm{SEs}$ for prevalence estimates were calculated using Taylor series linearisation; and prevalence estimates with a relative SE (RSE) considered potentially unreliable (RSE $>30 \%$ ) were not presented. ${ }^{6}$ Never and ever smokers were compared using two-tailed $t$ tests and differences were considered statistically significant at $\mathrm{p}<0.05$.

\section{RESULTS}

During 2007-2010, 4172 NHANES participants had valid spirometry, height and longest held occupation data, and were included in the study. The estimated age-standardised prevalence of airflow obstruction among ever-employed US adults aged 18-79 years was $13.7 \%$ (95\% CI $12.4 \%$ to $15.0 \%$ ) (table 1). The prevalence of airflow obstruction by demographic characteristic was highest in participants aged 60-79 years, males and nonHispanic whites. Among ever-employed adults reporting any dust/fumes occupational exposure, the prevalence of airflow obstruction was $14.3 \%$ (95\% CI $12.1 \%$ to $16.5 \%$ ). The occupation groups with a prevalence of airflow obstruction $>20 \%$ were installation, 
maintenance and repair occupations ( $\mathrm{p}=22.1 \%, 95 \% \mathrm{CI} 16.5 \%$ to $27.8 \%)$, and construction and extraction occupations ( $\mathrm{p}=20.7 \%, 95 \% \mathrm{CI} 13.5 \%$ to $27.9 \%)$. In contrast, office and administrative support occupations ( $\mathrm{p}=10.3 \%, 95 \%$ CI $6.2 \%$ to $14.5 \%$ ) had the lowest prevalence. The age-standardised prevalence of airflow obstruction by duration of occupational exposure to mineral dust, organic dust, exhaust fumes and other fumes was analysed but found not significantly different by duration of occupational exposure (quartiles) (results not shown).

Ever smokers had a significantly higher prevalence of airflow obstruction $(19.1 \%, 95 \% \mathrm{CI}$ $16.6 \%$ to $21.5 \%)$ compared with never smokers $(8.6 \%, 95 \%$ CI $7.0 \%$ to $10.1 \%)(\mathrm{p}<0.0001)$. The difference between prevalence estimates for never smokers and ever smokers remained significant for all demographic characteristics and occupational exposures analysed, with the exception of non-Hispanic black and Mexican American race/ethnicities. We estimated the prevalence of airflow obstruction by quartiles of pack-years for those aged 20-79 years (information on pack-years was not available for those $<20$ years) and found an increase in prevalence for ever smokers with 11 or more pack-years. The prevalence estimates of airflow obstruction were significantly higher among ever smokers compared with never smokers for construction and extraction occupations; food preparation and serving occupations; sales and related occupations; and office and administrative support occupations $(\mathrm{p}<0.05)$. However, prevalence estimates by cigarette smoking status were not comparable for all occupation groups due to potentially unreliable prevalence estimates.

\section{DISCUSSION}

We analysed NHANES data from 2007 to 2010 and estimated that the prevalence of spirometry-defined airflow obstruction among ever-employed US adults aged 18-79 years was $13.7 \%$. The prevalence was significantly higher among ever smokers (19.1\%) compared with never smokers (8.6\%). Among adults aged 60-79 years, the prevalence of airflow obstruction was $27.8 \%$ among ever smokers and $5.9 \%$ among never smokers. The prevalence of airflow obstruction for each occupational exposure was significantly higher among ever smokers compared with never smokers $(\mathrm{p}<0.05)$. This suggests an additive effect on airflow obstruction between smoking and occupational exposure, and is consistent with previous studies. ${ }^{10}$

The burden of airflow obstruction appeared to be disproportionally affecting workers in installation, maintenance and repair occupations and construction and extraction occupations. These occupation groups have known, sometimes complex, occupational exposures that include mixtures of gases, vapours, dust, fumes, sensitisers and other exposures. For example, workers in installation, maintenance and repair occupations can be exposed to fumes and sensitisers when soldering. ${ }^{11}$ Mechanics and service technicians repairing or replacing engines, or working on aircraft and vehicles, encounter fumes from diesel engines, as well as gases, vapours, sensitizers and metal dust exposures. ${ }^{11}$ Construction and extraction workers may be exposed to diesel from machinery combustion and also to organic and inorganic dusts, gases and fumes. ${ }^{11}$ Earth moving and extraction tasks performed by workers may result in exposure to coal mine dust, silica and other mineral dusts. ${ }^{11}$ 
A strength of our study is the use of population-based NHANES data on occupational exposure and longest held occupation. While this cross-sectional study has limitations for determining causality, longest held occupation data did provide information about the job that potentially contributed to the development of airflow obstruction. Industry group was available for longest held occupation, but we limited our analysis to occupation only for purposes of this short report. A limitation of this study is that no data were available to validate information on self-reported occupational exposure. Furthermore, use of reference equations developed from NHANES III data may lead to overestimation of the prevalence of airflow obstruction. ${ }^{1}$ Even by combining data from the NHANES 2007-2008 and 20092010 survey cycles to improve the reliability of prevalence estimates, the prevalence estimates for some occupation groups were unreliable. The inclusion of NHANES occupation data from the 2011 to 2012 survey cycle, once it is released, may help us compute reliable prevalence estimates for additional occupation groups.

\section{CONCLUSION}

The prevalence of spirometry-defined airflow obstruction among ever-employed US adults from 2007 to 2010 varied by demographic characteristics and occupational factors, and was generally higher among ever smokers regardless of demographic characteristics or occupational factors. Study findings emphasise the importance of monitoring the lung function of workers in occupations with a high prevalence of airflow obstruction, to help prevent or identify lung function decline. We recommend that future research be conducted and include NHANES occupation data from additional survey cycles. In addition, future industry-specific and occupation-specific studies are needed to identify specific occupations at an increased risk for airflow obstruction and to identify methods to reduce risk.

\section{Acknowledgments}

The authors would like to thank Megan Casey and Girija Syamlal, for providing helpful comments and critique of the manuscript; and Dr Lu-Ann Beeckman-Wagner for overseeing training, data collection and quality assurance of spirometry data.

Funding The National Institute for Occupational Safety and Health (NIOSH) supported the salaries of the authors. This work was performed by NIOSH employees as part of their work.

\section{REFERENCES}

1. Doney B, Hnizdo E, Dillon CF, et al. Prevalence of airflow obstruction in US adults aged 40-79 years: NHANES data 1988-1994 and 2007-2010. COPD. 2015; 12:355-365. [PubMed: 25244575]

2. Ryu JH, Scanlon PD. Obstructive lung diseases: COPD, asthma, and many imitators. Mayo Clin Proc. 2001; 76:1114-1153.

3. US Department of Health and Human Services. The health consequences of smoking - 50 years of progress: a report of the surgeon general. Atlanta, GA: Centers for Disease Control and Prevention, National Center for Chronic Disease Prevention and Health Promotion, Office on Smoking and Health; 2014. http://www.surgeongeneral.gov/library/reports/50-years-of-progress/index.html [accessed Oct 2015]

4. Balmes J, Becklake M, Blanc P, et al. American Thoracic Society statement: occupational contribution to the burden of airway disease. Am J Respir Crit Care Med. 2003; 167:787-797. [PubMed: 12598220] 
5. Hnizdo E, Sullivan PA, Bang KM, et al. Association between chronic obstructive pulmonary disease and employment by industry and occupation in the US population: a study of data from the Third National Health and Nutrition Examination Survey. Am J Epidemiol. 2002; 156:738-746. [PubMed: 12370162]

6. Centers for Disease Control and Prevention. National Health and Nutrition Examination Survey: analytic guidelines, 1999-2010. Hyattsville, MD: National Center for Health Statistics; 2013. (DHHS Publication No. 2013-1361). http://www.cdc.gov/nchs/data/series/sr_02/sr02_161.pdf [accessed Oct 2015]

7. Centers for Disease Control and Prevention. National Health and Nutrition Examination Survey Questionnaires, Datasets, and Related Documentation. Hyattsville, MD: National Center for Health Statistics; 2014. http://www.cdc.gov/nchs/nhanes/nhanes_questionnaires.htm [accessed Oct 2015]

8. Pellegrino R, Viegi G, Brusasco V, et al. Interpretative strategies for lung function testing. Eur Respir J. 2005; 26:948-968. [PubMed: 16264058]

9. Hankinson JL, Odencrantz JR, Fedan KB. Spirometric reference values from a sample of the general US population. Am J Respir Crit Care Med. 1999; 159:179-187. [PubMed: 9872837]

10. Blanc PD, Iribarren C, Trupin L, et al. Occupational exposures and the risk of COPD: dusty trades revisited. Thorax. 2009; 64:6-12. [PubMed: 18678700]

11. Stellman, JM. Encyclopedia of occupational safety and health. 4th. Geneva: International Labour Organization; 1998. 


\section{What this paper adds}

- Occupational exposures to vapours, gases, dust and fumes are associated with obstructive lung disease.

- Previous research suggests workers in specific occupations are more likely to have spirometry-defined chronic obstructive pulmonary disease, but the prevalence of spirometry-defined airflow obstruction among the 2007-2010 National Health and Nutrition Examination Survey ever-employed adult population is unknown.

- Prevalence of airflow obstruction varied by demographic characteristics and occupational factors and was higher among ever smokers than in never smokers.

- These findings support prior research on airflow obstruction and smoking, and emphasise the importance of monitoring the lung function of workers in occupations with a high prevalence of airflow obstruction. 


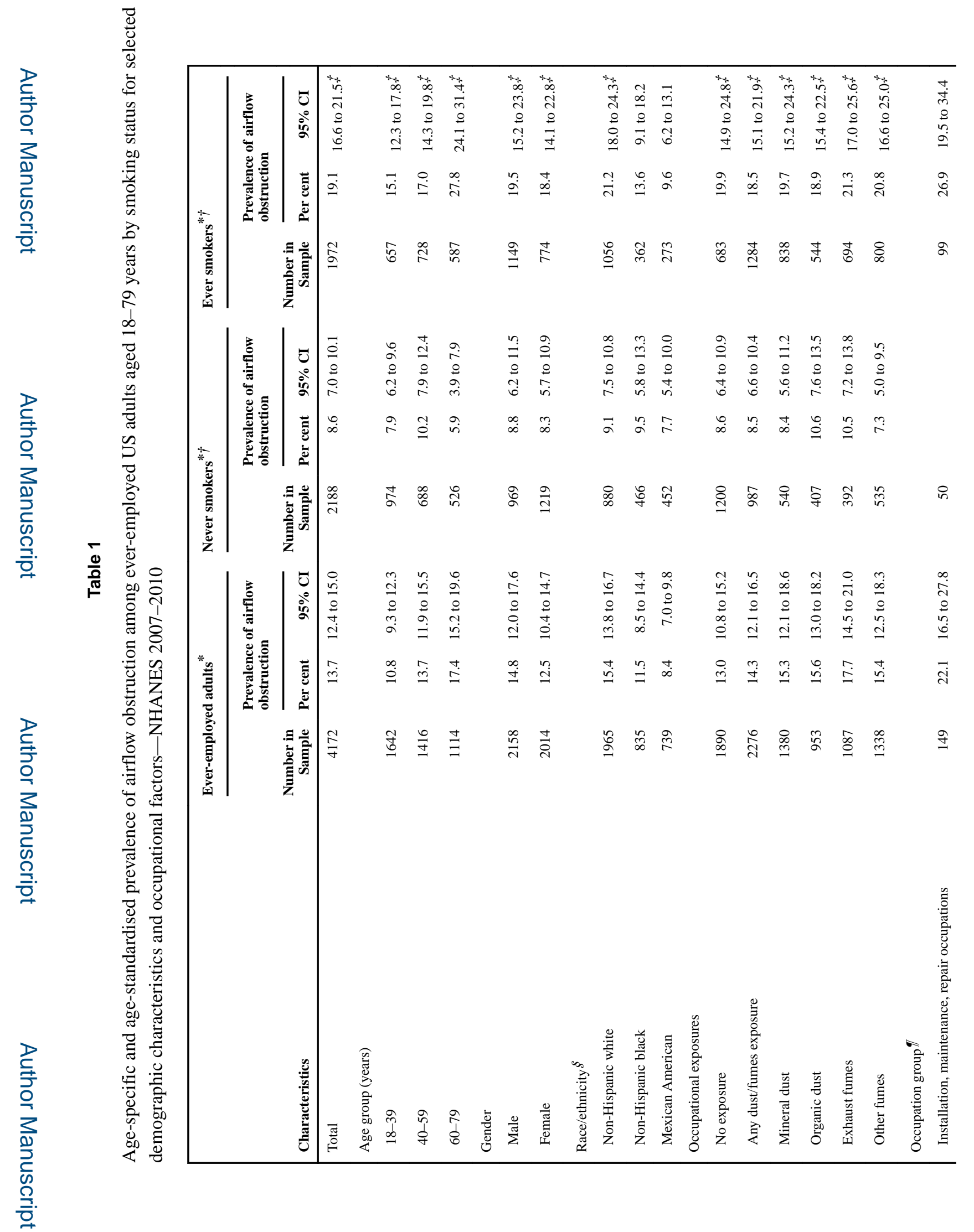

Occup Environ Med. Author manuscript; available in PMC 2017 March 15. 


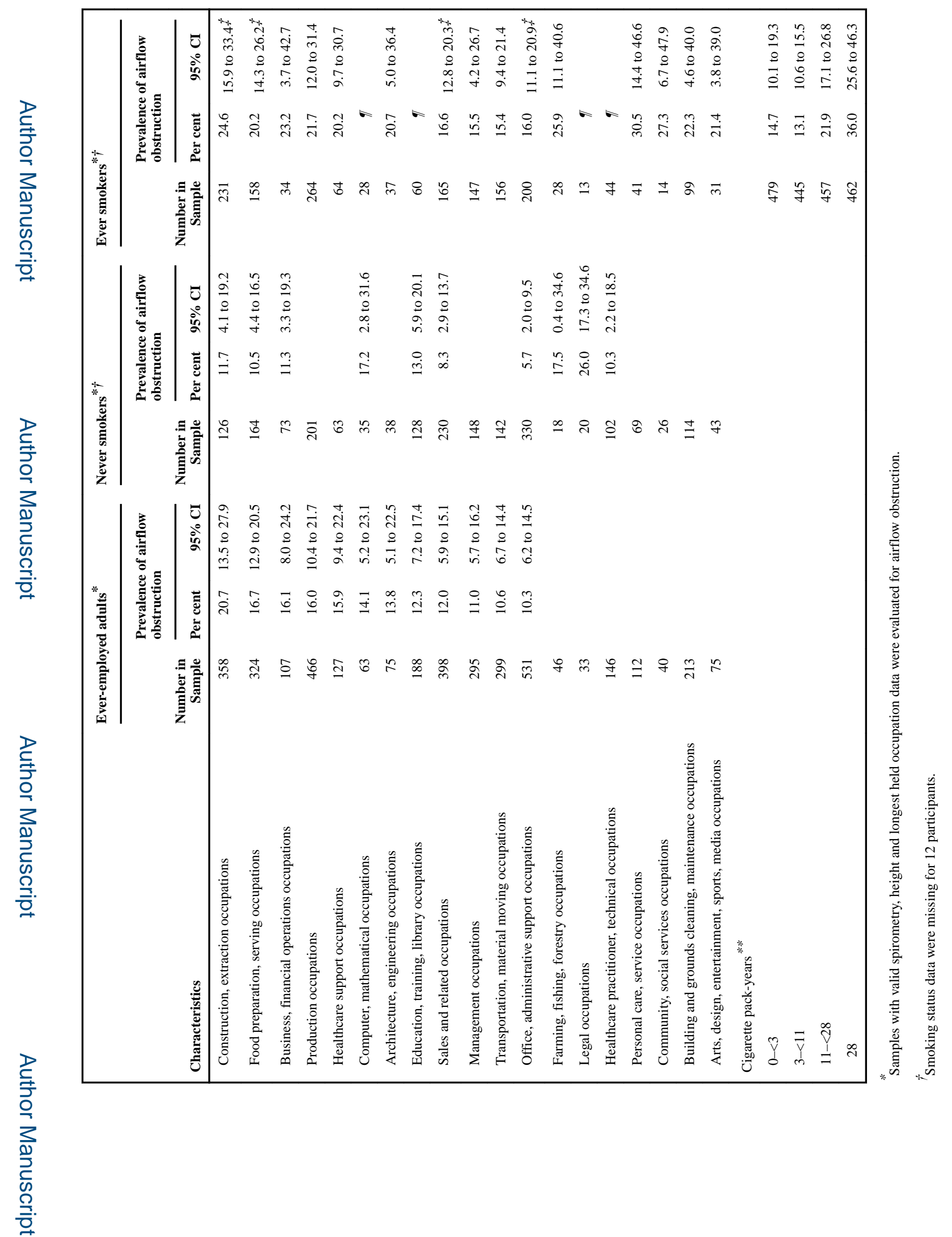

Occup Environ Med. Author manuscript; available in PMC 2017 March 15. 


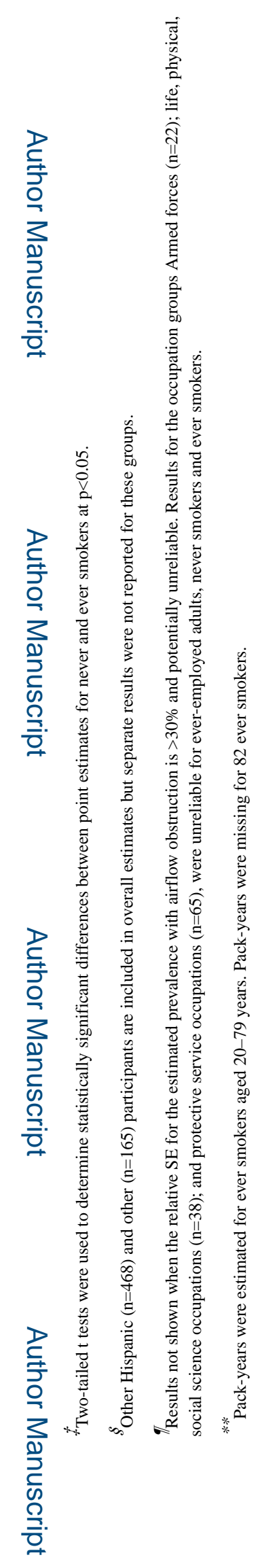

